\title{
Clinical advances in the management of chronic myelogenous leukemia: focus on bosutinib and patient considerations
}

This article was published in the following Dove Press journal:

Patient Preference and Adherence

8 July 2014

Number of times this article has been viewed

\author{
Kendra Sweet ${ }^{\prime}$ \\ Javier Pinilla-Ibarz' \\ Ling Zhang ${ }^{2}$ \\ 'Malignant Hematology, \\ ${ }^{2}$ Hematopathology, $\mathrm{H}$ Lee Moffitt \\ Cancer Center, Tampa, FL, USA
}

Correspondence: Kendra Sweet I 2902 Magnolia Dr, FOB3-Heme, Tampa, FL 336II, USA

Email kendra.sweet@moffitt.org

\begin{abstract}
The treatment for chronic myeloid leukemia has changed significantly over the past 15 years, and as of now, there are five BCR-ABL1 (breakpoint cluster region-Abelson murine leukemia viral oncogene homolog 1) tyrosine kinase inhibitors that have gained approval for treatment of this disease. All five are very effective drugs, and the decision surrounding which to use in specific patients is based on numerous factors. Bosutinib is one of the newer tyrosine kinase inhibitors to gain approval, and has been studied in the first-line setting as well as after failure of other tyrosine kinase inhibitors. It is an SRC-ABL1 (steroid receptor co-activator-ABL1) inhibitor that works in the presence of most kinase domain mutations. The primary side effects of bosutinib are gastrointestinal upsets. In the appropriate clinical setting, bosutinib can be considered a valuable addition to the armamentarium of treatments available for chronic myeloid leukemia.
\end{abstract}

Keywords: chronic myeloid leukemia, bosutinib, treatment options

\section{Introduction}

Chronic myeloid leukemia (CML) is a clonal myeloproliferative neoplasm characterized by the presence of the Philadelphia chromosome, which results from a reciprocal translocation between the breakpoint cluster region (BCR) of chromosome 22 and the Abelson murine leukemia viral oncogene homolog 1 (ABL1) region of chromosome 9. This $\mathrm{t}(9 ; 22)$ creates a fusion gene referred to as $B C R-A B L$, which then distributes itself throughout the cytoplasm of the cell, resulting in constitutive tyrosine kinase activity and development of CML. ${ }^{1}$ Epidemiologically, CML occurs at any age, and the peak age is in the 5th and 6th decades of life, with an annual incidence of 1-2 cases per 100,000 people. According to updated data from the American Cancer Society, CML accounts for $>10 \%$ of newly diagnosed adult leukemia cases in the USA annually. ${ }^{2}$ The prevalence of CML is increasing, and in 2014 there would be an estimated 160,000 people living with the disease. ${ }^{3}$

Historically, patients with CML were treated with hydroxyurea and interferon-alpha alone or in combination with low-dose cytarabine, and allogeneic stem cell transplantation was the only curative option. The treatment of CML changed dramatically in 2001 with the approval of imatinib (Gleevec ${ }^{\circledR}$; Novartis, Basel, Switzerland), the first BCR-ABL tyrosine kinase inhibitor (TKI). ${ }^{4,5}$ Since that time, three second-generation TKIs and one third-generation TKI have been released onto the market. Dasatinib (Sprycel $^{\circledR}$; Bristol-Myers-Squibb, New York, NY, USA) received approval for use after imatinib failure in 2006 and nilotinib (Tasigna ${ }^{\circledR}$; Novartis) received this indication in $2007.6,7$ Both of these second-generation TKIs received approval for first-line treatment of CML in 2010. ${ }^{8,9}$ In September 2012, the newest second-generation TKI, bosutinib 
(Bosulif $^{\circledR}$; Pfizer Inc., New York, NY, USA) came onto the market for use as a second-line agent in chronic phase, accelerated phase, or blast phase CML. ${ }^{10-12}$ In December of that year, the third-generation TKI, ponatinib (Iclusig ${ }^{\circledR}$; Ariad Pharmaceuticals Inc., Cambridge, MA, USA), was approved for use in CML patients who have failed at least one prior TKI. ${ }^{13,14}$ The indication for ponatinib recently changed due to safety concerns, and the drug is now used in the following situations: as second-line therapy or beyond when no other TKI is indicated, or when a T315I mutation is identified.

Following the rapid emergence of TKIs, it is critical for clinicians to understand how to best choose and apply them in different clinical settings. Although all the aforementioned TKIs are highly effective in the treatment of chronic phase CML, the need for multiple treatment options stems from the high rates of intolerance as well as resistance to different TKIs. Issues such as myelosuppression are seen across all of the TKIs listed above; however, each drug has a slightly different toxicity profile when analyzing nonhematologic toxicity. ${ }^{15}$ Furthermore, in CML patients who develop resistance to TKIs, the most common mechanism is the development of kinase domain mutations. ${ }^{16-18}$ Each TKI has slightly different efficacy in the setting of specific mutations. The T315I gatekeeper mutation has been the most elusive thus far, and ponatinib is the only effective TKI in patients with this mutation. ${ }^{13,14}$ The differences between each TKI are fairly clear; nonetheless, physicians face challenges when choosing which TKI is best for any particular patient. The side effects of each drug, patient comorbidities, and the results of a kinase domain mutation analysis are all factors that need to be taken into account. ${ }^{15}$

The current review focuses on bosutinib, one of the second-generation TKIs, and attempts to briefly summarize the characteristics of the drug in comparison with the other novel TKIs.

\section{Treatment options in CML}

As noted above, five BCR-ABL TKIs have been approved for the treatment of CML. Imatinib, dasatinib, and nilotinib all have an indication for first-line therapy in CML, and the decision surrounding which TKI is the most appropriate choice is based on a number of factors, including Sokal risk score at diagnosis, age, comorbidities, prescription cost, and physician preference. ${ }^{19}$

Anywhere from $15 \%-40 \%$ of patients will develop resistance or intolerance to their first-line TKI treatment, necessitating a change to a second-line TKI. ${ }^{5,8,9,15}$ Dasatinib and nilotinib also have an indication for use as second-line agents, and most experts would recommend these secondgeneration TKIs over imatinib in this setting. Imatinib is not typically considered as second-line therapy or beyond. ${ }^{19}$ At the time a patient displays resistance to first-line therapy, a kinase domain mutation analysis is recommended as per National Comprehensive Cancer Network guidelines. ${ }^{20}$ This is an essential component in selecting the most appropriate second-line TKI. ${ }^{21}$ Of importance, all TKIs have a different efficacy profile in the setting of specific mutations. For example, dasatinib tends to be ineffective in patients with a T315I, V299L, or F317L mutation, whereas those with a T315I, F359C/V, E255K/V, or Y253H mutation respond poorly to nilotinib. ${ }^{22-24}$

Bosutinib is the newest second-generation TKI, and can effectively treat patients with kinase domain mutations other than T315I and V299L. ${ }^{25,26}$ As opposed to bosutinib, the newest TKI, ponatinib, does not confer resistance with any known kinase domain mutations, and is the only one that produces a response in patients with T315I mutations. ${ }^{13,14}$ Initially, ponatinib was given a second-line indication in the treatment of CML; however, in October, 2013, it was temporarily removed from the market because of safety concerns. ${ }^{27}$ By January 2014, it returned to the market with a slightly different indication, stating it could be used in the setting of a T315I mutation or after failure of at least one TKI when no other TKI is indicated. ${ }^{28}$ In the appropriate patient population, ponatinib is a remarkably effective drug in CML, but must be used with caution given the known risks of arterial occlusive disease. In a Phase II trial with ponatinib, 9\% of patients experienced serious arterial thrombotic events. ${ }^{14}$

In addition, omacetaxine was also approved in late 2012 for use after failure of two or more TKIs. ${ }^{29}$ Omacetaxine is a protein translation inhibitor that has shown some efficacy in the setting of TKI failure as well as the presence of a T315I mutation. ${ }^{30}$

\section{Pharmacology of bosutinib}

Bosutinib (SKI606) is a dual BCR-ABL and SRC (steroid receptor co-activator)-ABL TKI. ${ }^{26,31,32}$ SRC family kinases have repeatedly been implicated in tumor progression in various solid tumors; however, emerging data support their role in BCR-ABL-independent forms of resistance and disease progression in CML as well. ${ }^{33-35}$

The chemical formula of bosutinib is $\mathrm{C}_{26} \mathrm{H}_{29} \mathrm{Cl}_{2} \mathrm{~N}_{5} \mathrm{O}_{3} \mathrm{H}_{2} \mathrm{O}$ and its molecular weight is $531 \mathrm{~g} / \mathrm{mol}$. It is a monohydrate 4-([2,4-dichloro-5-methylphenyl]amino)-6-methoxy-7-(3[4-methylpiperazin-1-yl]propoxy)quinolone-3-carbonitrile (Figure 1). ${ }^{25,26}$

Bosutinib is orally available and the recommended starting dose is $500 \mathrm{mg}$ daily, to be taken with food. It is highly protein-bound, with dose-dependent absorption. It is 


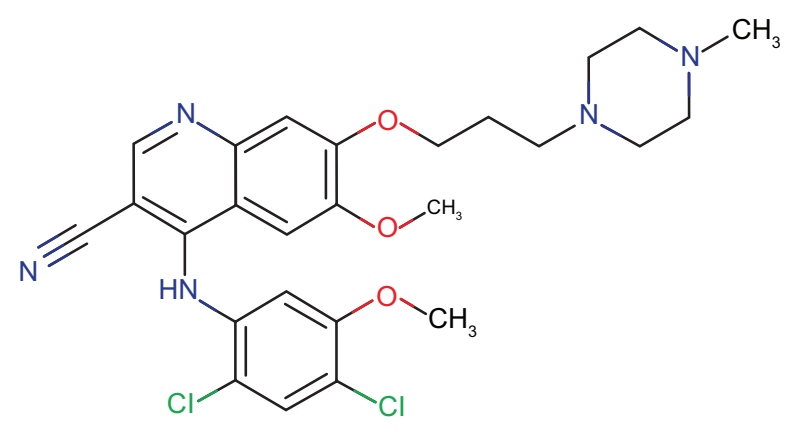

Figure I Structure of bosutinib.

primarily metabolized by the liver utilizing cytochrome $\mathrm{P} 450$ (CYP)3A4. ${ }^{25,36}$ Dose reduction is recommended in patients with hepatic dysfunction due to an increased frequency of QTe interval prolongation in this patient population. This potential side effect was not seen in those with normal liver function. ${ }^{37}$ Ninety-one percent of the drug is excreted in the feces and $3 \%$ in the urine..$^{25,26}$ Bosutinib exposure is impacted by coadministration with other CYP3A4 inducers or inhibitors, such as rifampin or ketoconazole, and these drugs should not be given concomitantly. ${ }^{25,26}$ Absorption is $\mathrm{pH}$-dependent; the solubility of bosutinib decreases as the $\mathrm{pH}$ in the stomach increases. Therefore, the use of proton pump inhibitors, such as lansoprazole, should be discontinued in anyone being treated with bosutinib. ${ }^{26,38-40}$ Patients requiring $\mathrm{pH}$-lowering therapy should be treated with antacids or histamine $\mathrm{H} 2$ receptor blockers taken at least 2 hours before or after bosutinib. ${ }^{37,41}$

After administration of bosutinib, the peak concentration of the drug occurs within 4-6 hours. ${ }^{26}$ The halflife is approximately 22 hours. ${ }^{26,37}$ A study looking at the pharmacokinetic-pharmacodynamic relationship of bosutinib found an exposure-response relationship for the incidence of diarrhea and a weak relationship for the incidence of rash. No other adverse events were found to have this relationship. Similarly, there was an exposureresponse relationship in newly diagnosed chronic phase CML patients for complete cytogenetic response, major molecular response, and cumulative complete hematologic response at 1 year; however, this relationship was not noted in patients who took bosutinib as a second-line or third-line agent. ${ }^{42}$

\section{Long-term efficacy, side effects, safety, and tolerability of bosutinib Efficacy}

The long-term efficacy of bosutinib has been studied in a Phase I/II trial analyzing the safety and efficacy of bosutinib in 288 patients with CML who were resistant or intolerant to imatinib. ${ }^{11}$ An additional 118 patients were later added to the trial, all of whom were resistant or intolerant to nilotinib or dasatinib. ${ }^{10}$ The recommended daily Phase II dose from this trial was $500 \mathrm{mg}$ orally. Of the patients with chronic phase CML who were resistant or intolerant to imatinib but had not received a second-generation TKI, the median follow-up was 24 months, and by this time point, $86 \%$ had achieved a complete hematologic response, $53 \%$ had achieved a major cytogenetic response, and $41 \%$ had achieved a complete cytogenetic response (CCyR). At the same time point, the progression-free survival for chronic phase CML patients was $79 \%$ and the overall survival was $92 \%$, which is comparable with other TKIs used in the second-line setting. ${ }^{11}$

The subgroup of 118 chronic phase CML patients who received more than one prior therapy before beginning treatment with bosutinib was studied independently. Median follow-up was 28.5 months, and rates of complete hematologic response, major cytogenetic response, and CCyR were $73 \%, 32 \%$, and $24 \%$, respectively. The 2 -year progression-free survival was $73 \%$ and overall survival was $83 \%$, indicating that bosutinib may be efficacious in this heavily pretreated patient population. Of the 118 patients evaluated, 37 were resistant to imatinib and dasatinib, 27 were resistant to imatinib and nilotinib, 50 were resistant to imatinib and intolerant of dasatinib, and four were resistant to imatinib, dasatinib, and nilotinib. Complete hematologic response was achieved in $50 \%, 67 \%$, and $77 \%$ of patients, respectively, in the group resistant to imatinib and dasatinib, the group resistant to imatinib and intolerant to dasatinib, and the group resistant to imatinib and nilotinib. In these same three cohorts, major cytogenetic response and CCyR rates were $31 \%, 30 \%$, and $35 \%$, and $14 \%, 28 \%$, and $27 \%$, respectively, indicating a trend toward lower response rates in patients who were resistant to dasatinib. ${ }^{10}$ Dasatinib is also an SRC/ABL inhibitor similar to bosutinib, which provides a potential explanation for this finding.

One hundred and thirty-four patients with advanced phase $\mathrm{CML}$ or $\mathrm{Ph}+$ acute lymphocytic leukemia were also studied. Of these, 63 had accelerated phase, 48 had blast phase, and $23 \mathrm{had} \mathrm{Ph}+$ acute lymphocytic leukemia. The median follow-up for all patients with advanced phase $\mathrm{CML}$ and $\mathrm{Ph}+$ acute lymphocytic leukemia was 8.3 months and a complete hematologic response was achieved in $61 \%$ of accelerated phase, $32 \%$ of blast phase, and $25 \%$ of $\mathrm{Ph}+$ acute lymphocytic leukemia patients. The overall complete hematologic response rate for the entire cohort was $47 \%$. Furthermore, $33 \%$ of accelerated phase, $29 \%$ of blast phase, and $100 \%$ of $\mathrm{Ph}+$ acute lymphocytic leukemia patients achieved a CCyR, 
with the overall CCyR rate being 34\%. Notably, only two patients from the $\mathrm{Ph}+$ acute lymphocytic leukemia cohort were evaluable for CCyR. Progression-free survival was 11.6 months for accelerated phase, 7.8 months for blast phase, and 2.7 months for $\mathrm{Ph}+$ acute lymphocytic leukemia patients. ${ }^{43}$

The efficacy of bosutinib in the setting of kinase domain mutations is another factor that makes it an enticing therapy in the second-line setting. In vitro half maximal inhibitory concentration $\left(\mathrm{IC}_{50}\right)$ values for bosutinib indicate efficacy in the setting of all ABL kinase domain mutations with the exception of T315I and V299L. ${ }^{44}$ All TKIs have different efficacy based on the mutations present, and the bosutinib profile is one that may make it useful in patients with mutations that are commonly resistant to dasatinib or nilotinib. ${ }^{25}$

Bosutinib has also been studied in the front-line setting in the BELA trial (ClinicalTrials.gov identifier NCT00574873). This study randomized 502 patients to receive either bosutinib $500 \mathrm{mg} /$ day orally or imatinib $400 \mathrm{mg} /$ day orally as first-line therapy. The primary end point was CCyR at 12 months. The study did not meet its primary end point, as CCyR was 70\% with bosutinib versus $68 \%$ with imatinib $(P=0.601)$, so bosutinib did not receive a US Food and Drug Administration (FDA) indication for first-line therapy. Despite the failure to meet its primary end point, this study found a much faster time to achieve CCyR in bosutinib-treated patients (12.9 weeks versus 24.6 weeks, $P \leq 0.001$ ). Moreover, the rate of major molecular response at 12 months and median time to major molecular response was significantly higher in patients on the bosutinib arm at $41 \%$ and 37.1 weeks, respectively, compared with only $27 \%$ and 72.3 weeks in the imatinib arm $(P \leq 0.001$ for both assessments). Transformation to accelerated phase or blast phase while on the study was lower on the bosutinib arm (2\% versus 4\%); however, this did not reach statistical significance. ${ }^{45}$ Some experts feel the reason this trial failed to meet its primary end point is because of the high rate of bosutinib discontinuation in the BELA study (19\% on bosutinib versus $6 \%$ on imatinib); however, it is possible that there are multiple factors that contributed to this finding, including lack of experience on the part of some investigators in dealing with the early side effects of the drug.

\section{Side effects, safety, and intolerance}

Bosutinib is an SRC-ABL inhibitor with a unique toxicity profile compared with the other approved BCR-ABL TKIs. Because it has fewer off-target effects, such as c-kit or platelet derived growth factor receptor (PDGFR), some of the toxicities seen with imatinib are not seen with bosutinib. ${ }^{12}$ The most frequent adverse events seen with bosutinib are gastrointestinal (diarrhea, nausea, vomiting) and rash, which tend to be mild, transient in nature, and respond to over the counter antidiarrhear medicine. ${ }^{11,45}$ Despite the relatively benign-appearing toxicity profile with bosutinib, it is worthwhile noting that $19 \%$ of patients in the BELA trial required discontinuation of the drug due to adverse events. This is compared with $6 \%$ in the imatinib arm. ${ }^{45}$

The safety and tolerability of bosutinib is consistent across all trials. The most common adverse event in all studies was diarrhea which occurred in up to $84 \%$ of patients; however, in the majority of these patients, the diarrhea was grade 1 or 2 . In the BELA trial, $11 \%$ of patients treated with imatinib experienced grade 3 or 4 diarrhea compared with only $1 \%$ on the imatinib arm. In the instances where bosutinib was interrupted due to diarrhea, this adverse event did not recur in the majority of patients when they were rechallenged. Importantly, although diarrhea led to $21 \%$ of patients requiring dose interruptions and $8 \%$ requiring dose reductions, it was not the cause of treatment discontinuation in any bosutinib-treated patient in the BELA trial. ${ }^{45}$ It did, however, lead to discontinuation in $2.5 \%$ of patients in the second-line and third-line trials. Other nonhematologic adverse events (all grades) occurring in $>20 \%$ of patients included nausea, vomiting, rash, pyrexia, abdominal pain, edema, fatigue, headache, and upper respiratory infections. ${ }^{10,11,43,45}$

Liver enzyme elevation was the most common laboratory adverse event in the second-line bosutinib trials, occurring in $10 \%-13 \%$ of all patients. Only $10 \%$ of these were grade 3 or $4 .{ }^{11}$ This was also a common adverse event in patients treated with bosutinib in the BELA trial, with grade 3 or 4 events occurring in $11 \%-22 \%$ of patients. Dose interruptions and reductions occurred in $57 \%$ and $36 \%$ of bosutinibtreated patients, respectively, compared with $24 \%$ and $6 \%$ in imatinib-treated patients. Two percent of bosutinib-treated patients required treatment discontinuation due to liver enzyme elevation. ${ }^{45}$

As with all BCR-ABL TKIs, myelosuppression is a common side effect of bosutinib. In the second-line studies, grade 3 or 4 thrombocytopenia, neutropenia, and anemia occurred in $25 \%, 19 \%$, and $8 \%$ of patients, respectively. The majority of these patients could be managed with dose reductions or dose interruptions. ${ }^{10,46}$ In the BELA trial, grade 3 or 4 thrombocytopenia, neutropenia, and anemia occurred in 14\%, 6\%, and $11 \%$ of bosutinib-treated patients, respectively. ${ }^{45}$ Again, because of the lack of c-kit inhibition by bosutinib, one would expect lower rates of myelosuppression with bosutinib when compared with other second-generation TKIs. ${ }^{12}$ In the DASISION trial (ClinicalTrials.gov identifier NCT00481247) that analyzed dasatinib as a first-line agent for CML, rates 
of grade 3 or 4 thrombocytopenia, neutropenia, and anemia were $19 \%, 21 \%$, and $10 \%$, respectively. ${ }^{47}$ Similarly, in the ENESTnd trial (ClinicalTrials.gov identifier NCT01564836) nilotinib was analyzed in the front-line setting, and rates of grade 3 or 4 thrombocytopenia, neutropenia, and anemia were $28 \%, 33 \%$, and $9 \%$, respectively, in patients treated with nilotinib $300 \mathrm{mg}$ orally twice daily. ${ }^{48}$

\section{Quality of life}

Now that CML is managed similarly to many other chronic illnesses, and most patients are expected to live for many years with their disease, quality of life becomes a pressing issue. Studies have shown that despite patients with CML having excellent responses to TKI therapy, they rate many factors related to quality of life much lower than their agematched and sex-matched controls who are free of a cancer diagnosis. ${ }^{49}$ Fatigue is a very common adverse event noted with all TKIs, that is rated worse in patients using TKI therapy for CML compared with normal controls. ${ }^{49,50}$

Patients from the Phase II and III trials with bosutinib were assessed for health-related quality of life. Trask et al provided a 44-item Functional Assessment of Cancer Therapy-Leukemia (FACT-Leu) questionnaire to patients on bosutinib for chronic phase CML who were either resistant or intolerant to imatinib. The patients were followed for 96 weeks, with the FACT-Leu being completed at baseline and again at weeks 36, 48 and 96. In both imatinib-resistant and imatinib-intolerant patients, the FACT-Leu assessments did not indicate significant impairment in most areas relating to health-related quality of life; however, there was improvement seen in emotional well-being over time. These improvements were significant in both groups of patients; however, they were only considered clinically meaningful in the patients classified as imatinib-intolerant. ${ }^{51}$

Notably, the 4-week assessment indicated a worsening of the physical well-being score in the group of patients considered imatinib-resistant and this was statistically significant. Investigators attributed this to side effects related to the initiation of bosutinib, and the scores improved over time. ${ }^{51}$

\section{Conclusion}

Management of CML has changed dramatically since the approval of imatinib in 2001, and patients with CML who have a complete cytogenetic response are expected to live a normal life span. ${ }^{52}$ However, there is still a significant percentage of patients who are resistant or intolerant to first-line treatment for CML and require a change in therapy. ${ }^{11}$ Overall, bosutinib is a valuable addition to the selection of BCR-ABL TKIs now approved for the treatment of CML.
It has good therapeutic efficacy in advanced phase CML and in those who are resistant or intolerant to dasatinib or nilotinib. Furthermore, the excellent tolerability of bosutinib makes it a very reasonable option for second-line therapy or beyond.

\section{Disclosure}

The authors report no conflicts of interest in this work.

\section{References}

1. Rowley JD. Letter: a new consistent chromosomal abnormality in chronic myelogenous leukaemia identified by quinacrine fluorescence and Giemsa staining. Nature. 1973;243(5405):290-293.

2. American Cancer Society. Cancer Facts \& Figures 2013. Available from: http://www.cancer.org/research/cancerfactsstatistics/cancerfactsfigures2013/index. Accessed May 20, 2014.

3. Huang X, Cortes J, Kantarjian H. Estimations of the increasing prevalence and plateau prevalence of chronic myeloid leukemia in the era of tyrosine kinase inhibitor therapy. Cancer. 2012;118(12):3123-3127.

4. O'Brien SG, Guilhot F, Larson RA, et al. Imatinib compared with interferon and low-dose cytarabine for newly diagnosed chronic-phase chronic myeloid leukemia. N Engl J Med. 2003;348(11):994-1004.

5. Druker BJ, Guilhot F, O'Brien SG, et al. Five-year follow-up of patients receiving imatinib for chronic myeloid leukemia. $N$ Engl $J$ Med. 2006;355(23):2408-2417.

6. Shah NP, Guilhot F, Cortes JE, et al. Long-term outcome with dasatinib after imatinib failure in chronic-phase chronic myeloid leukemia: follow-up of phase 3 study. Blood. 2014;123(15):2317-2324.

7. Kantarjian HM, Giles FJ, Bhalla KN, et al. Nilotinib is effective in patients with chronic myeloid leukemia in chronic phase after imatinib resistance or intolerance: 24-month follow-up results. Blood. 2011; 117(4):1141-1145.

8. Saglio G, Hochhaus A, Hughes TP, et al. ENESTnd update: nilotinib (NIL) vs imatinib (IM) in patients with newly diagnosed chronic myeloid leukemia in chronic phase (CML-CP) and the impact of early molecular response (EMR) and Sokal risk at diagnosis on long-term outcomes. Blood. 2013;122(21):92.

9. Hochhaus A, Kim DW, Shah NP, et al. Four-year follow-up of patients with newly diagnosed chronic myeloid leukemia in chronic phase (CML-CP) receiving dasatinib or imatinib: efficacy based on early response. Blood. 2013;122(21):653.

10. Khoury HJ, Cortes JE, Kantarjian HM, et al. Bosutinib is active in chronic phase chronic myeloid leukemia after imatinib and dasatinib and/or nilotinib therapy failure. Blood. 2012;119(15):3403-3412.

11. Cortes JE, Kantarjian HM, Brummendorf TH, et al. Safety and efficacy of bosutinib (SKI-606) in chronic phase Philadelphia chromosomepositive chronic myeloid leukemia patients with resistance or intolerance to imatinib. Blood. 2011;118(17):4567-4576.

12. Remsing Rix LL, Rix U, Colinge J, et al. Global target profile of the kinase inhibitor bosutinib in primary chronic myeloid leukemia cells. Leukemia. 2009;23(3):477-485.

13. Cortes JE, Kantarjian H, Shah NP, et al. Ponatinib in refractory Philadelphia chromosome-positive leukemias. $N$ Engl J Med. 2012;367(22):2075-2088.

14. Cortes JE, Kim DW, Pinilla-Ibarz J, et al. A Phase 2 trial of ponatinib in Philadelphia chromosome-positive leukemias. $N$ Engl J Med. 2013;369(19):1783-1796.

15. Khan AM, Bixby DL. BCR-ABL inhibitors: updates in the management of patients with chronic-phase chronic myeloid leukemia. Hematology. Epub 2013 December 2.

16. Jabbour E, Branford S, Saglio G, Jones D, Cortes JE, Kantarjian HM. Practical advice for determining the role of BCR-ABL mutations in guiding tyrosine kinase inhibitor therapy in patients with chronic myeloid leukemia. Cancer. 2011;117(9):1800-1811. 
17. Jabbour E, Jones D, Kantarjian HM, et al. Long-term outcome of patients with chronic myeloid leukemia treated with second-generation tyrosine kinase inhibitors after imatinib failure is predicted by the in vitro sensitivity of BCR-ABL kinase domain mutations. Blood. 2009;114(10):2037-2043.

18. Ernst T, Hochhaus A. Chronic myeloid leukemia: clinical impact of BCR-ABL1 mutations and other lesions associated with disease progression. Semin Oncol. 2012;39(1):58-66.

19. Baccarani M, Castagnetti F, Gugliotta G, Palandri F, Rosti G. Treatment recommendations for Chronic Myeloid Leukemia. Mediterr J Hematol Infect Dis. 2014;6(1):e2014005.

20. National Comprehensive Cancer Network. Chronic Myelogenous Leukemia version 3. 2014. Available from: http://www.nccn.org/about/news/ ebulletin/ebulletindetail.aspx?ebulletinid=277. Accessed May 21, 2014.

21. Radich JP. Monitoring response to tyrosine kinase inhibitor therapy, mutational analysis, and new treatment options in chronic myelogenous leukemia. J Natl Compr Canc Netw. 2013;11(5 Suppl):663-666.

22. Muller MC, Cortes JE, Kim DW, et al. Dasatinib treatment of chronicphase chronic myeloid leukemia: analysis of responses according to preexisting BCR-ABL mutations. Blood. 2009;114(24):4944-4953.

23. Cang S, Liu D. P-loop mutations and novel therapeutic approaches for imatinib failures in chronic myeloid leukemia. J Hematol Oncol. 2008; 1:15.

24. Hughes T, Saglio G, Branford S, et al. Impact of baseline BCR-ABL mutations on response to nilotinib in patients with chronic myeloid leukemia in chronic phase. J Clin Oncol. 2009;27(25):4204-4210.

25. Rusconi F, Piazza R, Vagge E, Gambacorti-Passerini C. Bosutinib: a review of preclinical and clinical studies in chronic myelogenous leukemia. Expert Opin Pharmacother. 2014;15(5):701-710.

26. Bethelmie-Bryan B, Lord K, Holloway S, Khoury HJ. Bosutinib treatment for Philadelphia chromosome-positive leukemias. Future Oncol. 2014;10(2):179-185.

27. Dalzell MD. Ponatinib pulled off market over safety issues. Manage Care. 2013;22(12):42-43

28. [No authors listed]. In brief: ponatinib (Inclusig) returns. Med Lett Drugs Ther. 2014;56(1434):8.

29. Alvandi F, Kwitkowski VE, Ko CW, et al. US Food and Drug Administration approval summary: omacetaxine mepesuccinate as treatment for chronic myeloid leukemia. Oncologist. 2014;19(1):94-99.

30. Cortes J, Lipton JH, Rea D, et al. Phase 2 study of subcutaneous omacetaxine mepesuccinate after TKI failure in patients with chronic-phase CML with T315I mutation. Blood. 2012;120(13):2573-2580.

31. Amsberg GK, Koschmieder S. Profile of bosutinib and its clinical potential in the treatment of chronic myeloid leukemia. Onco Targets Ther. 2013;6:99-106.

32. Levinson NM, Boxer SG. A conserved water-mediated hydrogen bond network defines bosutinib's kinase selectivity. Nat Chem Biol. 2014;10(2):127-132.

33. Summy JM, Gallick GE. Src family kinases in tumor progression and metastasis. Cancer Metastasis Rev. 2003;22(4):337-358.

34. Johnson FM, Gallick GE. SRC family nonreceptor tyrosine kinases as molecular targets for cancer therapy. Anticancer Agents Med Chem. 2007;7(6):651-659.

35. Li S. Src-family kinases in the development and therapy of Philadelphia chromosome-positive chronic myeloid leukemia and acute lymphoblastic leukemia. Leuk Lymphoma. 2008;49(1):19-26.
36. Stansfield L, Hughes TE, Walsh-Chocolaad TL. Bosutinib: a secondgeneration tyrosine kinase inhibitor for chronic myelogenous leukemia. Ann Pharmacother. 2013;47(12):1703-1711.

37. Syed YY, McCormack PL, Plosker GL. Bosutinib: a review of its use in patients with Philadelphia chromosome-positive chronic myelogenous leukemia. BioDrugs. 2014;28(1):107-120.

38. Abbas R, Hug BA, Leister C, Gaaloul ME, Chalon S, Sonnichsen D. A phase I ascending single-dose study of the safety, tolerability, and pharmacokinetics of bosutinib (SKI-606) in healthy adult subjects. Cancer Chemother Pharmacol. 2012;69(1):221-227.

39. Rassi FE, Khoury HJ. Bosutinib: a SRC-ABL tyrosine kinase inhibitor for treatment of chronic myeloid leukemia. Pharmgenomics Pers Med. 2013;6:57-62.

40. Tomilo DL, Smith PF, Ogundele AB, et al. Inhibition of atazanavir oral absorption by lansoprazole gastric acid suppression in healthy volunteers. Pharmacotherapy. 2006;26(3):341-346.

41. Pfizer, Bosulib. Prescribing information. Available from: http://labeling. pfizer.com/ShowLabeling.aspx?id=884. Accessed May 21, 2014.

42. Hsyu PH, Mould DR, Upton RN, Amantea M. Pharmacokineticpharmacodynamic relationship of bosutinib in patients with chronic phase chronic myeloid leukemia. Cancer Chemother Pharmacol. 2013; 71(1):209-218

43. Gambacorti-Passerini C, Cortes JE, Khoury HJ, et al. Safety and efficacy of bosutinib in patients with AP and BP CML and ph+ ALL following resistance/intolerance to imatinib and other TKIs: Update from study SKI-200. ASCO Meeting Abstracts. 2010;28(15 Suppl):6509.

44. Redaelli S, Piazza R, Rostagno R, et al. Activity of bosutinib, dasatinib, and nilotinib against 18 imatinib-resistant BCR/ABL mutants. J Clin Oncol. 2009;27(3):469-471.

45. Cortes JE, Kim DW, Kantarjian HM, et al. Bosutinib versus imatinib in newly diagnosed chronic-phase chronic myeloid leukemia: results from the BELA trial. J Clin Oncol. 2012;30(28):3486-3492.

46. Shen AQ, Wilson NM, Gleason SL, Khoury HJ. Bosutinib in the treatment of patients with Philadelphia chromosome-positive $(\mathrm{Ph}+)$ chronic myelogenous leukemia: an overview. Ther Adv Hematol. 2014;5(1):13-17.

47. Kantarjian H, Shah NP, Hochhaus A, et al. Dasatinib versus imatinib in newly diagnosed chronic-phase chronic myeloid leukemia. $N$ Engl J Med. 2010;362(24):2260-2270.

48. Saglio G, Kim DW, Issaragrisil S, et al. Nilotinib versus imatinib for newly diagnosed chronic myeloid leukemia. $N$ Engl J Med. 2010; 362(24):2251-2259.

49. Phillips KM, Pinilla-Ibarz J, Sotomayor E, et al. Quality of life outcomes in patients with chronic myeloid leukemia treated with tyrosine kinase inhibitors: a controlled comparison. Support Care Cancer. 2013;21(4): 1097-1103.

50. Pinilla-Ibarz J, Cortes J, Mauro MJ. Intolerance to tyrosine kinase inhibitors in chronic myeloid leukemia. Cancer. 2011;117(4):688-697.

51. Trask PC, Cella D, Besson N, Kelly V, Masszi T, Kim DW. Healthrelated quality of life of bosutinib (SKI-606) in imatinib-resistant or imatinib-intolerant chronic phase chronic myeloid leukemia. Leuk Res. 2012;36(4):438-442.

52. Gambacorti-Passerini C, Antolini L, Mahon FX, et al. Multicenter independent assessment of outcomes in chronic myeloid leukemia patients treated with imatinib. J Natl Cancer Inst. 2011;103(7):553-561.
Patient Preference and Adherence

\section{Publish your work in this journal}

Patient Preference and Adherence is an international, peer-reviewed, open access journal that focusing on the growing importance of patient preference and adherence throughout the therapeutic continuum. Patient satisfaction, acceptability, quality of life, compliance, persistence and their role in developing new therapeutic modalities and compounds to optimize
Dovepress

clinical outcomes for existing disease states are major areas of interest for the journal. This journal has been accepted for indexing on PubMed Central. The manuscript management system is completely online and includes a very quick and fair peer-review system, which is all easy to use. Visit http://www. dovepress.com/testimonials.php to read real quotes from published authors. 